\title{
Generalized Smoluchowski equation with correlation between clusters
}

\author{
Lionel Sittler \\ Theoretische Physik, Fachbereich 8, \\ Universität Wuppertal \\ and \\ Universität Duisburg-Essen, Germany
}

(Dated: November 11, 2018)

\begin{abstract}
In this paper we compute new reaction rates of the Smoluchowski equation which takes account of correlations. The new rate $K=K^{M F}+K^{C}$ are the sum of two terms. The first term is the known Smoluchowski rate with the mean-field approximation. The second takes account of a correlation between clusters. For this purpose we introduce the average path of a cluster. We relate the length of this path to the reaction rate of the Smoluchowski equation. We solve the implicit dependence between the average path and the density of clusters. We show that this correlation length is the same for all clusters. Our result depends strongly on the spatial dimension $d$.

The mean-field term $K_{i, j}^{M F}=\left(D_{i}+D_{j}\right)\left(r_{j}+r_{i}\right)^{d-2}$, which vanishes for $d=1$ and is valid up to logarithmic correction for $d=2$, is the usual rate found with the Smoluchowski model without correlation (where $r_{i}$ is the radius and $D_{i}$ the diffusion constant of the cluster). We compute a new rate: the correlation rate $K_{i, j}^{C}=\left(D_{i}+D_{j}\right)\left(r_{j}+r_{i}\right)^{d-1} M\left(\frac{d-1}{d_{f}}\right)$ is valid for $d \geq 1$ (where $M(\alpha)=\sum_{i=1}^{+\infty} i^{\alpha} N_{i}$ is the moment of the density of clusters and $d_{f}$ is the fractal dimension of the cluster). The result is valid for a large class of diffusion processes and mass-radius relations. This approach confirms some analytical solutions in $d=1$ found with other methods. We show also Monte-Carlo simulations which illustrate some exact new solvable models.
\end{abstract}


There are different microscopic processes which lead to the model with the Smoluchowski equation. A comprehensive review can be found in [1] and the derivation of the reaction rate can be found in [2] with the mean-field approach. Particles can be injected into the system during time evolution [3415667], the injection of particles can be periodic in time [819]. For a very anisotropic surface the model is practically one-dimensional [10111]. Hence we will assume the most general model for an arbitrary space dimension $d$ and flux $F(t)$ of particles injected into the system. Our model has two properties:

1. Brownian diffusion: clusters of mass $k$ diffuse with a Brownian motion with a diffusion constant $D_{k}$.

2. irreversible aggregation: clusters interact through a contact process, namely two particles in contact aggregate irreversibly.

The Smoluchowski approach disregards the microscopic density $n_{k}(t, \mathbf{r})$ of a cluster of mass $k$ and considers the macroscopic density, i.e. space average density: $N_{k}(t)=$ $\frac{1}{\int d^{(d)} \mathbf{r}} \int d^{(d)} \mathbf{r} n(t, \mathbf{r})$. The Smoluchowski equation for the density of a cluster is:

$$
\frac{d N_{k}}{d t}=\frac{1}{2} \sum_{i+j=k} K_{i, j} N_{i} N_{j}-N_{k} \sum_{i=1}^{+\infty} K_{i, k} N_{i}+F(t) \delta_{k, 1},
$$

where $K_{i, j}$ is the reaction rate and $\delta_{i, j}$ the Kronecker symbol. We assume the following initial conditions: if there is no source $F(t)=0$ we have $N_{i}(t=0)=\delta_{1, i}$, otherwise with a source we have $N_{i}(t=0)=0$. A useful quantity is the moment of order q:

$$
M_{q}(t)=\sum_{i=1}^{+\infty} i^{q} N_{i} .
$$

The time evolution of the moment is:

$$
\dot{M}_{q}(t)=\frac{1}{2} \sum_{i, j} K_{i, j}\left((i+j)^{q}-i^{q}-j^{q}\right) N_{i} N_{j}+F(t) .
$$

For $q=1$ the moment is the total coverage $M_{1}$, i.e. the total mass, and for $q=0$ it is the total cluster density $M_{0}$. Without source the total mass is conserved $M_{1}(t)=M_{1}(0)=N_{1}(0)=1$. The calculation of the reaction rate depends on some physical assumptions The mean-field theory assumes we can exchange the many-body problem by a one-body problem with a convenient external field. For this purpose we define asymmetric rates, the rate that a cluster 
of mass $i$ aggregates with the cluster $j: K_{i \rightarrow j}$ (the reaction rate is a symmetric function of $K_{i \rightarrow j}$ ). Following [12], [10] (we chose a characteristic length instead of a characteristic lifetime used in [10]) and dimension analysis, we define the average length of a cluster with the following equation:

$$
\frac{\partial N_{i}}{\partial t} \sim-\frac{D_{i} N_{i}}{\xi_{i}^{2}}=-N_{i} \sum_{j=0}^{+\infty} K_{i \rightarrow j} N_{j}
$$

the life-time $\tau_{i}$ of the cluster is defined with the relation $\xi_{i}=\sqrt{D_{i} \tau_{i}}[10]$. We follow the mean-field approach [2]; we chose an arbitrary particle $n_{i}(\mathbf{r}, t)$ and the microscopic equation of the cluster reads [12]:

$$
\frac{N_{i}-n_{i}}{\xi^{2}}=\Delta^{(d)} n_{i}
$$

where $\Delta^{(d)}$ is the symmetrical Laplace operator in $d$ dimensions:

$$
\Delta^{(d)}=\frac{\partial^{2}}{\partial r^{2}}+\frac{d-1}{r} \frac{\partial}{\partial r} .
$$

The fluctuations $\left(N_{i}-n_{i}\right)$ around the average value $N_{i}$ are proportional to the variation of the microscopic density $\Delta^{(d)} n_{i}$. We consider a spherically symmetric solution of Eq. (3). The external field is the same as for the mean-field approach [2], the boundary conditions $n_{i}(r \rightarrow+\infty)=N_{i}$ and $n\left(r=r_{j}\right)=0$ express the convenient external field. Physically it means that close to a cluster the microscopic density vanishes, i.e we have a perfect sink and far away from a sink the microscopic density is close to the average density (we assume an early stage of clustering). The stationary spherically symmetric solution of the Eq. (3) ( we chose as origin the contact between the cluster $i$ and the point-like cluster $j$ ) reads [13]:

$$
n_{i}(r)=\left\{\begin{aligned}
N_{i}\left(1-\exp \left(-\frac{r-r_{j}}{\xi_{i}}\right)\right) & \text { for } d=1 \\
N_{i}\left(1-\frac{K_{0}\left(\frac{r}{\xi_{i}}\right)}{K_{0}\left(\frac{r_{j}}{\xi_{i}}\right)}\right) & \text { for } d=2 \\
N_{i}\left(1-\frac{r_{j} \exp \left(-\frac{r-r_{j}}{\xi_{i}}\right)}{r}\right) & \text { for } d=3
\end{aligned}\right\}
$$

where $K_{0}$ is the modified Bessel function [14]. The reaction rates is proportional to the flux of cluster $i$ into a cluster $j$ [2] $\left.\int_{j} d \mathbf{r}_{j} \frac{D_{i}}{N_{i}} \frac{\partial n_{i}}{\partial r}\right|_{r=r_{j}}$, then:

$$
K_{i \rightarrow j}=\left\{\begin{aligned}
\frac{D_{i}}{\xi_{i}} & \text { for } d=1 \\
\frac{D_{i} r_{j}}{\xi_{i}} \frac{K_{1}\left(\frac{r_{j}}{\xi_{i}}\right)}{K_{0}\left(\frac{r_{j}}{\xi_{i}}\right)} \sim D_{i}\left(1+\frac{r_{j}}{\xi_{i}}\right) & \text { for } d=2 \\
D_{i}\left(r_{j}+\frac{r_{j}^{2}}{\xi_{i}}\right) & \text { for } d=3
\end{aligned}\right\}
$$


We have used the asymptotic expansion of the modified Bessel Function $\frac{x K_{1}(x)}{K_{0}(x)} \sim 1+x$ for $x \rightarrow+\infty[14]$ ( on length scale of the order $\xi_{i} \ll r_{j}$, i.e. $x \rightarrow 0$ we have a logarithmic behavior $\frac{x K_{1}(x)}{K_{0}(x)} \sim-\frac{1}{\ln (x / 2)}$ which is typical at the critical dimension $d=2$ ).

The rate for all dimensions is formally:

$$
K_{i \rightarrow j}=D_{i}\left(r_{j}^{d-2}+\frac{r_{j}^{d-1}}{\xi_{i}}\right) .
$$

Eq. (4) contains two terms. The first term $r_{j}^{d-2}$, which is (strictly speaking) valid only for $d \geq 3$, we call the mean-field term. The second term which is valid only for $d \geq 2$, contains the correlation, implicitly defined in $\xi_{i}$, we call the correlation term. Eq. (4) is also valid for $d=1$ if we put $r_{j}^{d-2}=0$. One then combines the Eq. (4) with Eq. (2), we get an equation of the second order on $\xi_{i}$ :

$$
\xi_{i}^{2} \sum_{j} r_{j}^{d-2} N_{j}+\xi_{i} \sum_{j} r_{j}^{d-1} N_{j}-1=0
$$

The approximate solution, for a large time scale where the correlation length is short, i.e $\xi_{i} \ll r_{j}$, is

$$
\xi_{i}=\frac{1}{\sum_{j} r_{j}^{d-1} N_{j}}
$$

Notice that the average length $\xi_{i}$ is independent of the mass $i$. It is on average the inverse of an effective coverage

$$
\sum_{j} r_{j}^{d-1} N_{j}
$$

and the life-time $\tau_{i}=\frac{\xi_{i}^{2}}{D_{i}}$ depends on the mass $i$. We obtain the asymmetric rate:

$$
K_{i \rightarrow j}=D_{i}\left(r_{j}^{d-2}+r_{j}^{d-1} \sum_{k=1}^{+\infty} r_{k}^{d-1} N_{k}\right)
$$

The symmetrization of the reaction rate [2] leads to:

$$
K_{i, j}=\left(D_{i}+D_{j}\right)\left(\left(r_{j}+r_{i}\right)^{d-2}+\left(r_{i}+r_{j}\right)^{d-1} \sum_{k=1}^{+\infty} r_{k}^{d-1} N_{k}\right)
$$

One generally assumes that the gyration radius scales with the mass $r_{i}=i^{\alpha}$ ( in appropriate length units), where $\alpha=1 / d_{f}$ is the inverse of the fractal dimension of the boundary of the cluster( for compact island $d_{f}=d-1$, and $d_{f}=1.72$ for Diffusion Limited Aggregation process in in $d=2[12])$. The effective coverage is for compact islands, $M_{(d-1) / d_{f}}$ is close to 
the coverage $M_{1}$ when $d \gg 1$.

From the Eq. (6) we define two rates: the mean-field rate $K_{i, j}^{M F}=\left(D_{i}+D_{j}\right)\left(r_{j}+r_{i}\right)^{d-2}$ and the rate with correlation $K_{i, j}^{C}=\left(D_{i}+D_{j}\right)\left(r_{j}+r_{i}\right)^{d-1} M_{\alpha(d-1)}$. The difference between these both rates is that we exchange a spatial dimension $\left(r_{j}+r_{i}\right)$ in mean-field rate through a moment $M_{\alpha(d-1)}$ in the rate with correlation. We have hence a Taylor expansion around the correlation number $\frac{M_{\alpha(d-1)}}{\xi_{i}}$. In $d=1$ only the correlation term remains and the rate is:

$$
K_{i, j}=\left(D_{i}+D_{j}\right) M_{o}
$$

We assume $M_{\alpha(d-1)} \rightarrow M_{0}$ when $d \rightarrow 1$, because the scaling effect of the radius of gyration vanishes in $d=1$, i.e. the boundary for an aggregating particle is point-like.

For a time scale larger than $t_{c}$, defined by $M_{\alpha(d-1)}\left(t_{c}\right) \sim\left(r_{i}+r_{j}\right)$, the correlations dominate, hence the reaction rate is essentially:

$$
K_{i, j}=K_{i, j}^{C}=\left(D_{i}+D_{j}\right)\left(r_{j}+r_{i}\right)^{d-1} M_{\alpha(d-1)}
$$

We can derive solutions of the Smoluchowski equation with only rate with correlation Eq. (8) from known solutions with mean-field rate $K_{i, j}^{M F}=\left(D_{i}+D_{j}\right)\left(r_{j}+r_{i}\right)^{d-2}$ in a special case, without source $(F=0)$ and with a rate given from Eq. (8) the solution of the Smoluchowski equation in $d$ dimensions is equivalent to the solution with the mean-field rate $K^{M F}$ without source $(F=0)$, up to a time rescaling $d \tilde{t}=M_{\alpha(d-1)}(t) d t$, in $d+1$ dimensions.

With our method a large class of processes in $d=1$ can be solved analytically.

In order to confirm this approach we present some analytical solutions and numerical evidence.

1. The solution of the Smoluchowski equation with $K_{i, j}=1$ and without source $(F=0)$ is known analytically [2], $N_{k} \sim t^{-2}$ and $M_{0} \sim t^{-1}$, hence the time rescaling $d \tilde{t}=M_{0}(t) d t$ leads us to the known solution in $d=1$ of the clustering process with mass-independent diffusion. This solution was found with another method [18] is $M_{0} \sim t^{-1 / 2}$. Notice that through our method we obtain the asymptotic behavior for all clusters, $N_{k} \sim t^{-1}$ ( FIG.1) which was not possible with the method used in [18].

2. With constant source $F=$ const, and $D_{i}=1$ we have the following equations: 


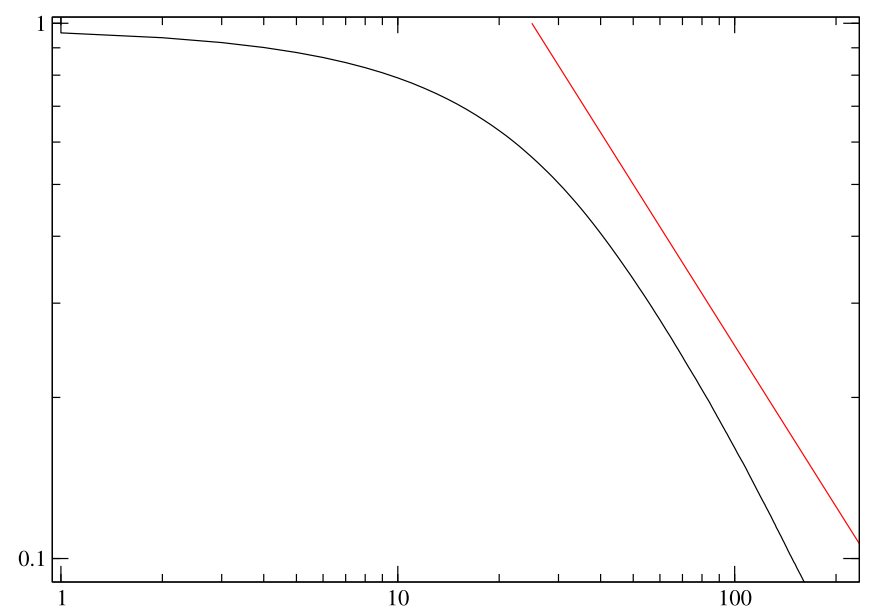

FIG. 1: Cluster density $N_{1}$ as a function of time (for comparison the red curve is $\sim t^{-1}$ )

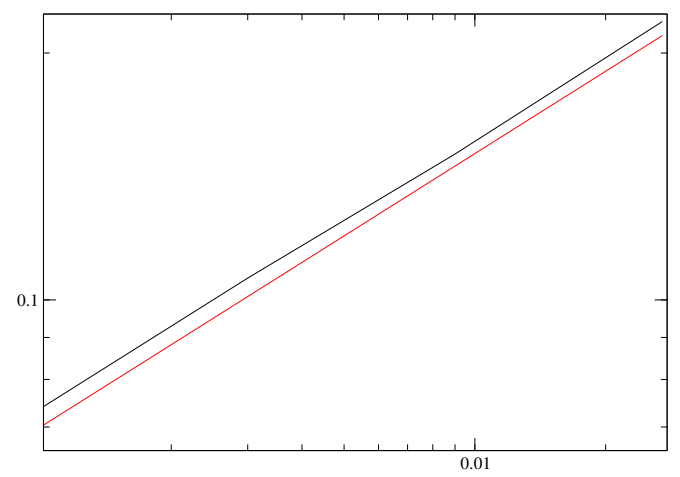

FIG. 2: Asymptotic value of the total cluster density $M_{0}$ as a function of the flux $F$ (for comparison the red line has a slope $F^{1 / 3}$ )

$$
\dot{N}_{k}=M_{0} \sum_{i+j=k} N_{i} N_{j}-2 N_{k} M_{0}^{2}+\delta_{1, k} F \text { and } \dot{M}_{0}=-M_{0}^{3}+F
$$

The asymptotic solutions are $M_{0} \sim F^{\frac{1}{3}}$ ( FIG.2) and $N_{k} \sim k^{-\frac{3}{2}}$ ( FIG.3). For comparison the solution with the mean-field term [15]16] in $d=2$ is $N \sim F^{\frac{1}{2}}$ and $N_{k} \sim k^{-3 / 2}$

3. For the sake of completeness we present an analytical solution already known: $D_{k}=$ $\delta_{1, k}$ and source $F=$ const. The solutions were found with another method in [10] 


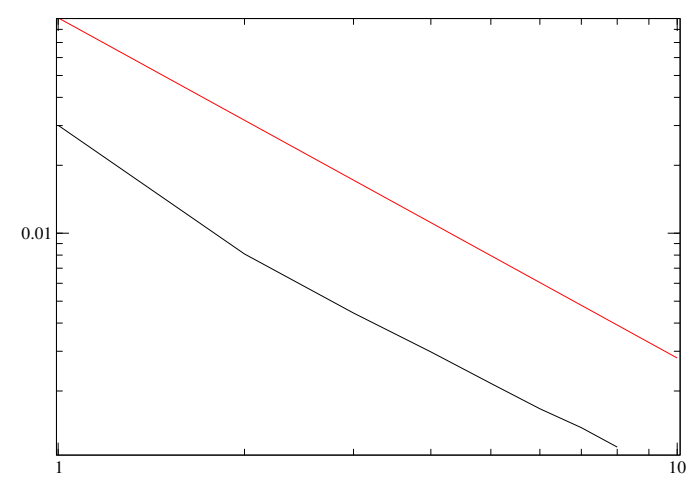

FIG. 3: Asymptotic value of the cluster density $N_{k}$ as function of the cluster mass $k$ (for comparison the straight line $\sim k^{-3 / 2}$ )

are $N_{1} \sim t^{-1 / 2}$ and total cluster density $M_{0} \sim t^{1 / 4}$. With our method we obtain the same rates and therefore the same solution. For comparison the mean-field approach gives $N_{1} \sim t^{-1 / 3}$ and $M_{0} \sim t^{1 / 3}$ which are different to the numerical solution and the experimental result.

Hence we have shown that this approach leads to the correct description of irreversible aggregation with brownian diffusion in one dimension for different models. We hope to find similar results in $d>1$ beyond the time scale where the mean-field rate is valid, i.e. for $t>t_{c}$.

There are some open questions:

1. Higher order of the Taylor expansion: can we compute other rates when the correlation between cluster is more important, i.e. can we obtain the full Taylor expansion around the correlation parameter $\frac{M_{\alpha(d-1)}}{\xi_{i}}$ Perhaps larger correlation effect can be obtained by modifying the boundary condition of Eq. (3)

2. multiple particles reaction: how can this approach be generalized to systems where the binding energy is so tight that binary reaction is negligible and we have to consider the reaction $A_{1}+\cdots+A_{n} \rightarrow B_{1}+\cdots+B_{m}[19]$ with an arbitrary $n$ and $m$ ? 
* I would like to thanks D.E.Wolf for introducing me to the papers [10]12]

1 M.H. Ernst, in Fractal in Physics, 289 ed. by L.Pietronero and E. Tosatti, North-Holland, Amsterdam (1986),

2 S.K. Friedlander, Smoke, Dust and Haze, Oxford, University Press, New York, (2000), 2nd ed.,

3 J.G Amar, F. Family and P.-M. Lam, Phys. Rev. B50, 8781(1998),

4 W. Matthew, Epitaxial Growth, (Academic, New York, 1975)J.Y. Tao, Materials Fundamentals of Molecular Beam Epitaxy, (World Scientific, singapore, 1993),

5 L.H. Tang, J. Phys. I 3, 935 (1993),

${ }^{6}$ F.Westerhoff, L. Brendel and D.E. Wolf, in Structure and Dynamics of heterogeneous Systems, edited by P.Entel and D.E. Wolf ( World Scientific, singapore, 2000),

7 A.-L. Barabàsi and H.E. Stanley,Fractal concepts in surface growth Cambridge University Press (1995),

8 D.B.Chrisey. and G.K. Hubler,Pulsed Laser Deposition of Thin Films (Wiley, New York, 1994),

9 P.Jensen and B. Niemeyer,Surface science384,L823-L827 (1997),

10 A. Pimpinelli, J. Vilain, and D.E. Wolf Phys. Rev. Lett. , 69 985, (1992),

11 Y.W. Mo, J. Kleiner, M.B. webb, and Lagally, Phys. Rev. Lett., 66, 1998, (1991),

12 G.S. Bales and D.C. Chrzan, Phys. Rev. B, 50 6057(1994),

13 R. Dautray- J.-L. Lions, Analyse mathématique et calcul numérique, 2 Masson (1987),

14 M. Abramowitz and I.A. Stegun Handbook of mathematical functions, Dover publications, Inc. $(1972)$,

15 P.L Krapivsky, J.F.F Mendes, and S.Redner, Phys. Rev. B, 59 15950-15958 (1999),

16 P.L Krapivsky, J.F.F Mendes, and S.Redner, Eur. Phys. J. B, 4 401-404 (1998),

17 N.V. Brilliantov and P.L. Krapivsky, J. Phys. A: Math.Gen., 24, 4787-4803 (1991),

18 H.Hinrichsen, Ad. in Phys., 49,815-958(2000),

19 A.A. Naidenov and S.K. Nechaev cond-mat/0209271. 